\title{
Optimization Method of Integrated Light-Screen Array with External Parameters Based on Genetic Algorithm
}

\author{
Rui Chen (D), BoWen Ji, Ding Chen, and ChenXi Duan \\ School of Optoelectronic Engineering, Xi'an Technological University, Shaanxi, Xi'an 710032, China \\ Correspondence should be addressed to Rui Chen; chenrui_xatu@163.com
}

Received 30 July 2021; Accepted 25 August 2021; Published 11 September 2021

Academic Editor: Thippa Reddy G

Copyright (C) 2021 Rui Chen et al. This is an open access article distributed under the Creative Commons Attribution License, which permits unrestricted use, distribution, and reproduction in any medium, provided the original work is properly cited.

\begin{abstract}
Due to the high sensitivity and fast response, the light-screen array measurement principle is suitable for the dynamic parameter measurement of small and fast targets including projectile. Since the spatial structures of the light-screen array determine the measurement accuracy, internal parameters such as the angles between the light-screens are usually calibrated and then directly used in the field. However, the effect of the measuring state is ignored in the test field. This paper takes the integrated light-screen array sky vertical target as the research object, and two rotation angles are introduced as external parameters to describe the deviation between the calibration state and measuring state of the target, so as to optimize the measurement model. Aiming at the problem that the external parameters cannot be measured directly, an external parameter inversion method of machine learning based on a genetic algorithm is designed under a complex engineering model. The deviation between the projectile hole and the light-screen array measurement coordinates is used to build an inversion database for the genetic algorithm during the machine learning process. The simulation and the live firing test show that the optimization method and parameter identification algorithm in this paper can optimize the measurement model and improve the measurement accuracy of the light-screen array principle directly and can also provide a reference for the optimization and parameter identification in other engineering problems.
\end{abstract}

\section{Introduction}

The flight parameters of some small moving targets, such as projectile, bullet, sport ball, and miniature unmanned aerial vehicle, attract much interest in many related fields. For example, it can help athletes improve their competitive performance, and it can also make the missions more smoothly completed for miniature unmanned aerial vehicles. Particularly, the measurement of projectile flight parameters is a highly concerning issue in the process of weapon development and production all over the world $[1,2]$.

The current measurement methods are mainly based on the acoustic principle [3], radar principle [4], CCD intersection principle [5], and light-screen array principle [6, 7]. Due to the fact that the acoustic principle cannot apply to subsonic projectiles, the radar principle is susceptible to electromagnetic interference, and the CCD intersection principle is expensive and difficult to operate, compared with other principles, the light-screen array principle has many advantages of high sensitivity, greater range of velocity measurement, fast response, and easy operation [8], and it can meet the measurement requirements of different characteristics of projectiles emitted by different types of barrel weapons, such as subsonic weapons, small minor-caliber weapons, and rapid-fire weapons, so it is necessary to improve its performance.

The measurement accuracy of the light-screen array depends on the precision of the description of the lightscreen spatial structure $[9,10]$. At present, the most effective method to obtain these structural parameters is calibration [11]. However, due to the influence factors such as environmental and human factors, the calibration state is difficult to be accurately reproduced in the test field [12], which leads to the structure deviation of the light-screen array; hence, the measurement model is inconsistent with the reality and the measurement accuracy is reduced. More importantly, the structure deviation is difficult to be directly measured due to the lack of reference, so they are ignored in the test field. 
Therefore, research on model optimization and the method to identify the key parameters of the optimized model is necessary.

In recent years, big data has generated strong interest in various scientific and engineering domains over the last few years, which not only is being widely used in the field of healthcare, production, sales, IoT devices, Web, organizations, etc., but also plays an obvious role in the field of engineering, such as data analysis, calculation model parameter acquisition, and intelligent algorithm optimization [13]. And various algorithms have been applied to solve problems in these complex engineering fields, such as an advancing coupled multistable stochastic resonance method being realized, which considers the signal-to-noise ratio as the fitness function to optimize and determine the system parameters, so that the faults of motor bearings are detected in the field of engineering rotating machinery [14], using the cooperative coevolution framework simplifying the algorithm process by dividing the largescale and high-dimensional complex optimization problem into several lowdimensional optimization subproblems [15] and using an improved quantum-inspired cooperative coevolution algorithm based on combining the strategies of cooperative coevolution to optimize the airport gate allocation method and realized to effectively allocate airport gates to the flights [16].

In this paper, the parameters in the current measurement model are defined as internal parameters, and the external parameters from influence factors in the test field are introduced to improve the measurement model; furthermore, the inversion algorithm based on genetic algorithm is studied. The proposed algorithm establishes the objective function through the inversion idea; after the data dimension is reduced, the external parameters are acquired from the shooting database by machine learning based on genetic algorithm in reproduction. Comparing with the current light-screen parameter calibration method, the parameter acquisition does not depend on special measurement experiments, so it is more applicable. Finally, the method is verified by simulation and live firing tests, and the results of this paper can be directly used to optimize the measurement model and improve the measurement accuracy of photoelectric detection, and the algorithm can also provide a reference for the identification of model parameters in other engineering problems.

\section{Measurement Principle and System Parameters}

2.1. Measurement Principle and Internal Parameters. The basic measurement principle of the light-screen array is to form a specific shape (such as double $\mathrm{N}$ shape, double $\mathrm{V}$ shape, and double parallel shape) of the light-screen array in space by arranging the light-screens. After shooting, the time of the projectile arriving at each light-screen can be recorded; then, combined with the structure of the lightscreen array, the flight parameters of velocity and coordinates can be calculated.
The method of light-screen generating equipment is basically the same. Take the typical sky screen vertical target as an example, it consists of a lens, a slit, a photodetector, etc., and forms the light-screens of a certain shape in the space above it. For example, the double N-shaped lightscreen array is composed of two single $\mathrm{N}$-shaped vertical targets, as shown in Figure 1.

In Figure 1, in order to constrain the light-screen structure, target I and target II are fixed on the pedestal after adjusting and aligning by the laser to form the integrated light-screen array. The trajectory direction is set as the $X$ -axis; then, the system of coordinate $O X Y Z$ is established, and the projection view of the light-screen structure is shown in Figure 2.

The structure parameters of the light-screen array can be described by the vertical angles between light-screen planes $\alpha=\left[\alpha_{1}, \alpha_{2}, \alpha_{3}, \alpha_{4}\right]$ and the horizontal angles between lightscreen planes $\beta=\left[\beta_{1}, \beta_{2}\right]$. And $s_{1}$ is the distance between target I and target II. The current measurement model is fully described by these parameters above, which are collectively referred to as internal parameters in this paper.

The structure description of the double N-shaped lightscreen array is shown in

$$
A \cdot I=B
$$

where

$$
A=\left[\begin{array}{ccc}
\cos \alpha_{1} & \sin \alpha_{1} & 0 \\
\cos \beta_{1} & 0 & \sin \beta_{1} \\
\cos \alpha_{2} & -\sin \alpha_{2} & 0 \\
\cos \alpha_{3} & \sin \alpha_{3} & 0 \\
\cos \beta_{2} & 0 & \sin \beta_{2} \\
\cos \alpha_{4} & -\sin \alpha_{4} & 0
\end{array}\right],
$$

$$
B=\left[\begin{array}{c}
0 \\
0 \\
0 \\
s_{1} \cdot a_{1} \\
s_{1} \cdot a_{2} \\
s_{1} \cdot a_{3}
\end{array}\right] \text {. }
$$

The projectile flight velocity and the coordinates obtained can be expressed as follows in the measurement coordinate system $O X Y Z$ by ignoring the gravity and air resistance:

$$
X=M^{-1} \cdot N
$$




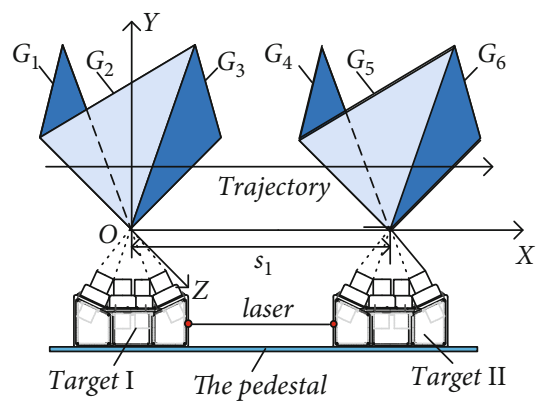

FIGURE 1: Measurement principle of double $\mathrm{N}$ shape light-screen array.
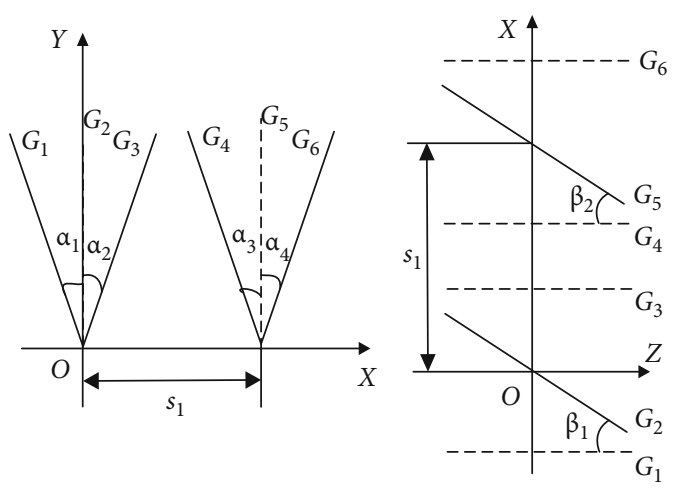

FIGURE 2: Projection view of light-screen and internal parameters.

where

$$
\begin{gathered}
M=\left[\begin{array}{cccccc}
\cos \alpha_{1} & \sin \alpha_{1} & 0 & 0 & 0 & 0 \\
\cos \beta_{1} & 0 & \sin \beta_{1} & \cos \beta_{1} \cdot t_{2} & 0 & \sin \beta_{1} \cdot t_{2} \\
\cos \alpha_{2} & -\sin \alpha_{2} & 0 & \cos \alpha_{2} \cdot t_{3} & -\sin \alpha_{2} \cdot t_{3} & 0 \\
\cos \alpha_{3} & \sin \alpha_{3} & 0 & \cos \alpha_{3} \cdot t_{4} & \sin \alpha_{3} \cdot t_{4} & 0 \\
\cos \beta_{2} & 0 & \sin \beta_{2} & \cos \beta_{2} \cdot t_{5} & 0 & \sin \beta_{2} \cdot t_{5} \\
\cos \alpha_{4} & -\sin \alpha_{4} & 0 & \cos \alpha_{4} \cdot t_{6} & -\sin \alpha_{4} \cdot t_{6} & 0
\end{array}\right], \\
X=\left[\begin{array}{c}
x_{1} \\
y_{1} \\
z_{1} \\
v_{x 1} \\
v_{y 1} \\
v_{z 1}
\end{array}\right], \\
0 \\
0 \\
N=\left[\begin{array}{c}
0 \\
0 \\
s_{1} \cos \alpha_{3} \\
s_{1} \cos \beta_{2} \\
s_{1} \cos \alpha_{4}
\end{array}\right] .
\end{gathered}
$$

The internal parameters of light-screen angles $\left[\alpha_{1}, \alpha_{2}\right.$, $\left.\alpha_{3}, \alpha_{4}\right],\left[\beta_{1}, \beta_{2}\right]$, and target distance $s_{1}$ are all constant values, which can be obtained by the calibration. Time series $t_{i}(i$ $=1,2,3, \cdots, 6)$ indicates the time when a projectile arrives at each light-screen, respectively, which can be obtained by the chronograph.

2.2. External Parameters and Model Optimization. It is obvious that the structure of the light-screen array directly affects the measurement accuracy. However, it is difficult for the vertical target to reproduce its calibration state in the test field, and the influence is mainly manifested in Figure 3.

In Figure 3, the vertical target is not completely horizontal after placement in the test field, which causes the entire light-screen array to rotate angle $\tau$ along the $Z$-axis (as shown in Figure 3(a) and angle $\omega$ along the $X$-axis (as shown in Figure 3(b). These two angles are mutually independent, since they are orthogonally decomposed. The whole structure of the light-screen array is changed by these two angles which are called external parameters in this paper.

The measurement model is optimized by introducing external parameters into the measurement model shown in Formula (1), which is closer to the measuring state, as shown in

$$
\begin{gathered}
A \cdot I_{\tau}=B, \\
A \cdot I_{\omega}=B,
\end{gathered}
$$

where

$$
\begin{aligned}
I_{\tau} & =\left[\begin{array}{ccc}
\cos \tau & \sin \tau & 0 \\
-\sin \tau & \cos \tau & 0 \\
0 & 0 & 1
\end{array}\right] \cdot I, \\
I_{\omega} & =\left[\begin{array}{ccc}
1 & 0 & 0 \\
0 & \cos \omega & \sin \omega \\
0 & -\sin \omega & \cos \omega
\end{array}\right] \cdot I .
\end{aligned}
$$

It is noteworthy that the external parameters are often ignored because they are difficult to directly measure in the test field. The external parameter inversion method of machine learning based on genetic algorithm is hereby studied.

\section{Inversion Model and Simulation}

Since there is no physical contact between the light-screen array and the projectile, a cardboard target is placed perpendicular to the trajectory direction after the vertical target; then, the coordinates of the projectile can be measured by the light-screen array and the cardboard target, simultaneously. The coordinates of the projectile hole on the cardboard target can be regarded as a quasitruth value, as shown in Figure 4.

The projectile coordinates $(z, y)$ are measured by the light-screen array and $(z *, y *)$ is the coordinates of projectile hole on the cardboard target plane. In Figure $4, s_{2}$ is the distance between target II and the cardboard target, so that the coordinates can be inferred from the light-screen plane 


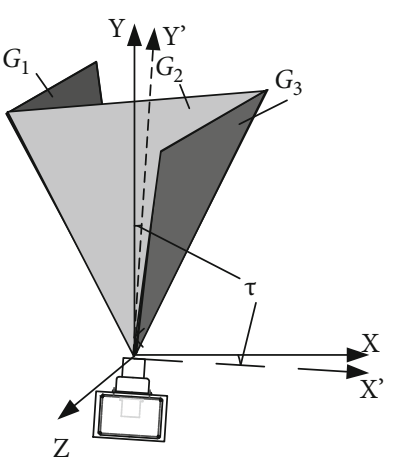

(a) Rotate along the $Z$-axis

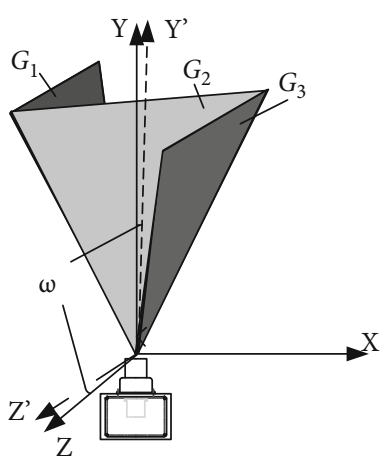

(b) Rotate along the $X$-axis

FIgURE 3: The changes of light-screen array structure in measuring state.

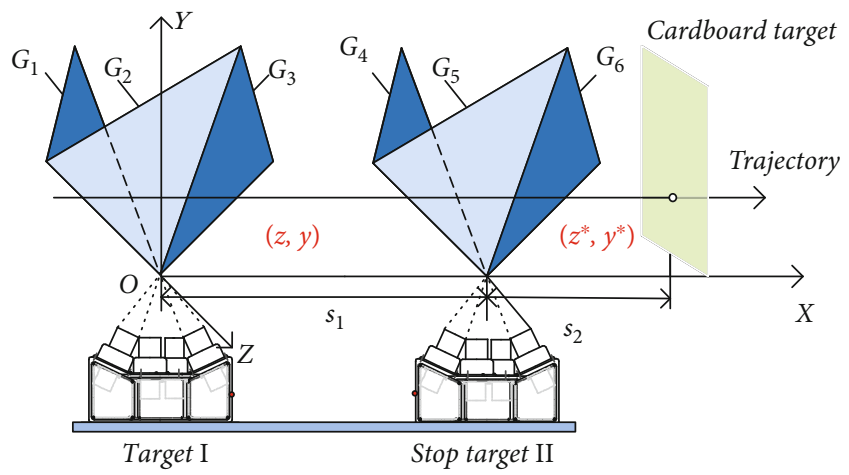

FIGURE 4: Structure parameter inversion model of light-screen array.

to the cardboard target plane via the flight parameters measured by the light-screen array. The simplified expression shown in Formula (8) causes the expression to be complicated to expand:

$$
\left\{\begin{array}{l}
z=f\left(\tau, \omega, s_{i n}, t_{n}, s_{2}\right) \\
y=g\left(\tau, \omega, s_{i n}, t_{n}, s_{2}\right)
\end{array}\right.
$$

In Formula (8), symbol $s_{\text {in }}$ represents the internal parameters, symbol $t_{n}(n=1,2,3, \cdots, 6)$ represents the time series of projectile arrival at each light-screen plane, and $\tau$ and $\omega$ are the external parameters to be inverted.

In the ideal case, the coordinates $(z, y)$ which are measured by the light-screen array should equal the projectile hole coordinates $(z *, y *)$ on the cardboard target plane. However, due to the influence of the external parameters and other factors, there is always some deviation between them, which represents the closeness between the measured coordinates of the light-screen array and the quasitruth value.

The optimization model contains many parameters and has a complex influence mechanism on the measurement results. Although some of the parameters are related to the results, they still can be regarded as constant values in the measurement process. These constant values have no effect on the coordinate deviation, so ignoring the influence of these constant values can reduce the dimension of the inversion parameters and reduce the algorithm burden [17].

Under the condition that other influencing factors remain unchanged, the larger the value of external parameters, the larger the deformation of the light-screen, and the larger the coordinate deviation of $(z, y)$ and $(z *, y *)$. The objective function is constructed by considering the deviation of horizontal and vertical coordinates comprehensively, which is shown as

$\delta=\sum_{k=1}^{j} \frac{1}{j}\left(\left(f_{k}\left(\tau, \omega, s_{i n}, t_{n}\right)-z_{k}^{*}\right)^{2}+\left(g_{k}\left(\tau, \omega, s_{i n}, t_{n}\right)-y_{k}^{*}\right)^{2}\right)^{1 / 2}$.

In order to reduce the influence of random error, the data obtained from multiple shots were used to establish the inversion database, which can be used to build a blockchain to provide services for obtaining model parameters in the algorithm below. Symbols $j$ is the total number of projectiles involved in inversion, and $k$ is the projectile serial number.

The parameters in the engineering model are decomposed according to the attributes, and the external parameters are taken as the parameters to be retrieved; then, the blockchain constructed according to the objective function serves as the inversion database in the genetic algorithm. Parameter values of the external parameter are inverted by machine learning based on a genetic algorithm.

Due to the random error being ignored and other conditions remaining unchanged, the data of blockchain is only affected by external parameters. The initial population with various characteristics is established within the range of external parameter values, and the population is reproduced by a genetic algorithm. In the breeding process, the objective function value can be calculated for each population value in the blockchain, and the population with a smaller objective function has a greater chance of survival. When the objective function is the smallest, the external parameter values are closest to their true values. The process of the inversion method of machine learning based on a genetic algorithm can be expressed in Figure 5. 


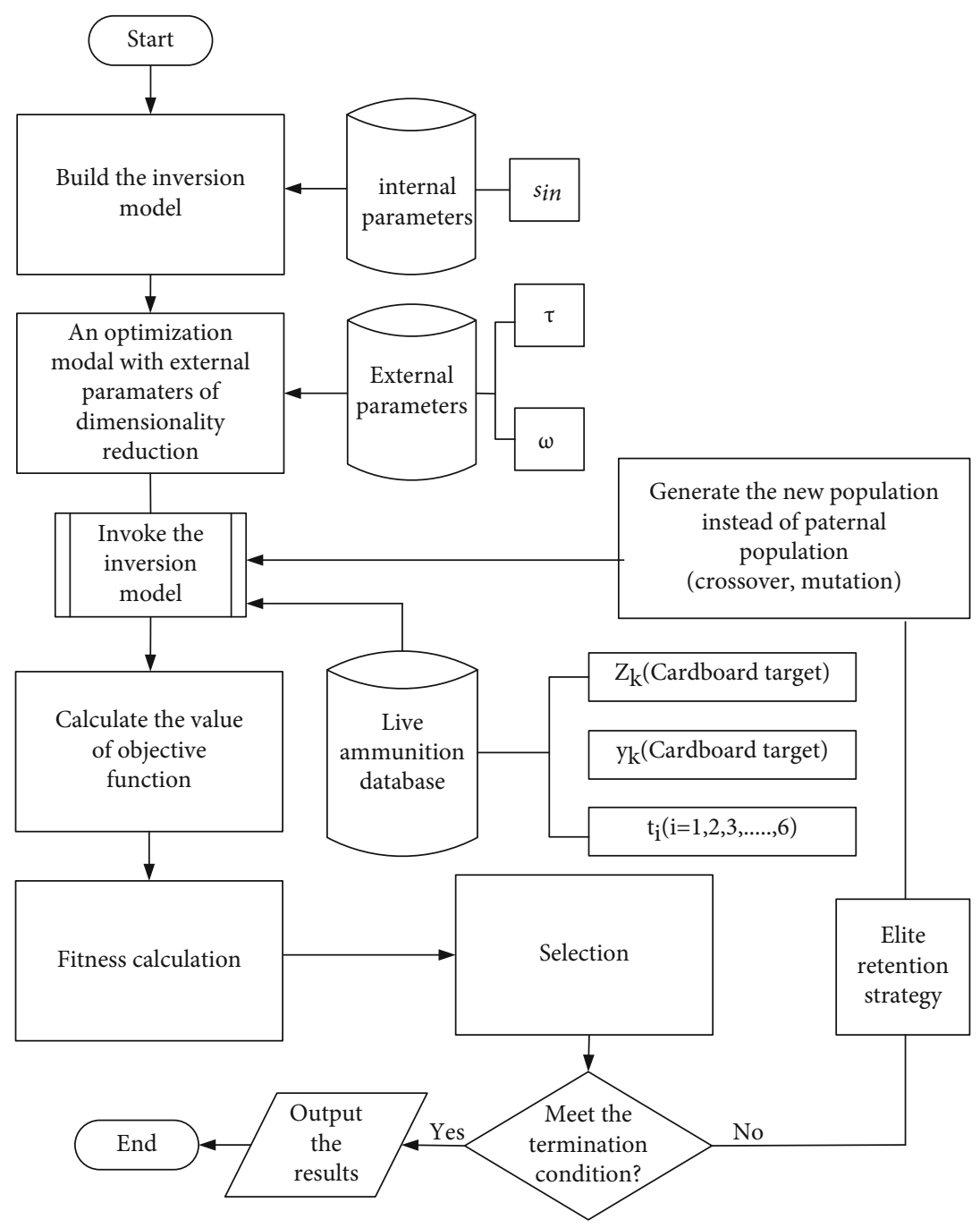

FIGURE 5: The diagram of machine learning based on genetic algorithm.

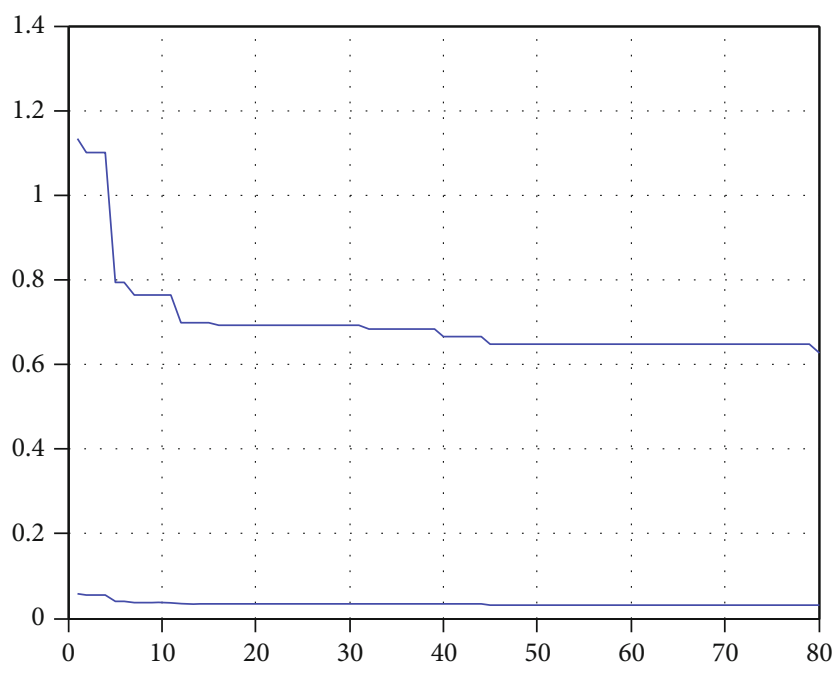

FIgURE 6: Simulation of genetic algorithm inversion method.
TABLe 1: The external parameters obtained by simulation.

\begin{tabular}{lcc}
\hline \multirow{2}{*}{ The value types } & \multicolumn{2}{c}{ External parameters } \\
& $\tau\left({ }^{\circ}\right)$ & $\omega\left(^{\circ}\right)$ \\
\hline Set values & 0.15 & 0.30 \\
Initial values & 0 & 0 \\
Inverse values & 0.18 & 0.26 \\
\hline
\end{tabular}

TABLE 2: Experimental result of live firing.

\begin{tabular}{lcc}
\hline \multirow{2}{*}{ The value types } & \multicolumn{2}{c}{ External parameters } \\
& $\tau\left({ }^{\circ}\right)$ & $\omega\left(^{\circ}\right)$ \\
\hline Initial value & 0 & 0 \\
Inverse value & 0.05 & 0.09 \\
\hline
\end{tabular}

The measurement model is optimized by introducing external parameters, and the model parameter dimension is reduced by inputting all the calibrated internal parameters and ignoring the constant parameters. Then, focus on the external parameters in which the initial value and value 


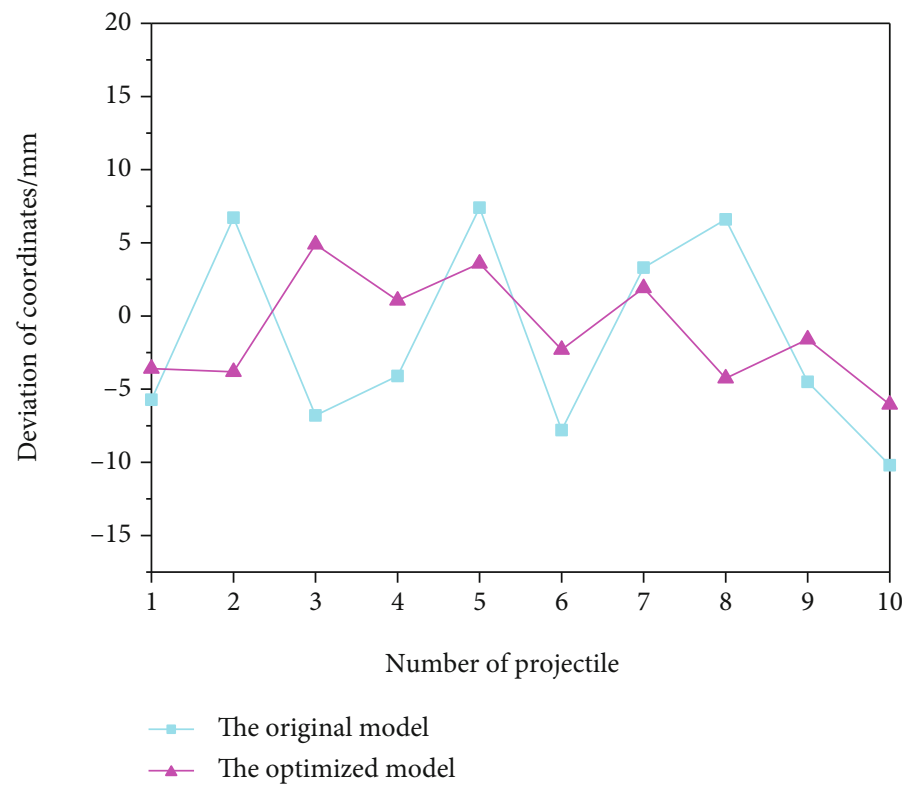

Figure 7: Distribution of coordinate deviation.

range are constraint in the test range. The constraint conditions are expressed as

$$
\left\{\begin{array}{l}
\tau=\tau_{0} \pm \sigma_{\tau}, \\
\omega=\omega_{0} \pm \sigma_{\omega} .
\end{array}\right.
$$

The shooting data is obtained by generating random numbers with the above range by simulation and using the objective function to build the blockchain. The fitness function is obtained on the basis of blockchain in the genetic algorithm section, and the elite strategy is introduced in the competition process to improve efficiency. The population gene with the small value of the objective function has a higher fitness and selected to be inherited to the next generation with a higher probability. When the objective function is less than a certain threshold and the generation number reaches the threshold value, the algorithm ends and the final inversion result is given.

The inversion model of the double N-shaped lightscreen array was established in MATLAB, and the internal parameters are input according to the measured value of the field test; then, the value range of external parameters is limited to $\tau \in\left[-0.5^{\circ}, 0.5^{\circ}\right]$ and $\omega \in\left[-0.8^{\circ}, 0.8^{\circ}\right]$.

Take $j=20$ times shot within the $500 \mathrm{~mm} \times 500 \mathrm{~mm}$ rectangular target surface to establish the database to obtain the time series $t_{i}$ and target coordinates for simulation. Considering the diversity of the population and the amount of program calculation, the population number is set to 20 . Take 2 significant digits to conduct binary coding according to

$$
2^{m_{j}-1}<(a-b) \times 10^{n}<2^{m_{j}}-1 .
$$

In Formula (11), $b$ and $a$ are the upper and lower bounds of the angle values, $n$ is the precision requirement, and $m_{j}$ is the length of the encoding string.

Start the MATLAB general toolbox and enter the genetic algorithm. The key parameters such as crossover probability are $85 \%$, and mutation probability is $20 \%$ in the genetic algorithm, and set the reproduction generation $\mathrm{GEN}=80$ as a condition for the termination. The result is shown in Figure 6.

During the simulation process, the coordinate deviation of $(z, y)$ and $(z *, y *)$ is gradually reduced, and the efficiency of the inversion algorithm is obviously decreased with the increase of the reproduction algebra and gradually tends to be stable.

The simulation results are compared with the set values, and the results are shown in Table 1.

It can be seen from Table 1 that the external parameters after simulation are closer to the set values.

\section{Verification Experiment}

In order to verify the external parameter inversion method, the experiment is designed and carried out with live firing; then, these shooting data were used to modify the blockchain and participate in machine learning algorithms instead of simulation data. After the light-screen array vertical target is well arranged, the internal parameters are inputted and the measurement model is established; then, the external parameter measurement model is introduced for optimization, and the value range is estimated which is within the simulation value range.

After random shots on the $500 \mathrm{~mm} \times 500 \mathrm{~mm}$ rectangular target surface, the database of the time series $t_{i}$ and target coordinates is obtained. The external parameters are learned by the machine learning, and the external parameters of the optimized model are identified. The experimental data is shown in Table 2. 
After the optimization model is identified, 10 random shots are performed again. The coordinates of these projectiles are calculated separately under the original model and the optimized model; then, the calculated results are compared with the projectile hole coordinates on the cardboard target. The result is shown in Figure 7.

The experiment shows the deviation between cardboard target and the light-screen array measurement resulting under two different models which both fluctuate around zero. But the deviation of measurement resulting under an optimized model is smaller, which means that it is closer to the true value. The optimization model and the inversion method of obtaining external parameters are feasible, which makes the light-screen array have higher precision.

\section{Conclusions}

In order to effectively improve the measurement accuracy of the light-screen array principle, this paper proposes an optimized measurement model by introducing external parameters, whose spatial structure is more close to the actual measured state. Then, focusing on the problem of external parameter identification, the inversion method of machine learning based on genetic algorithm is further studied. The objective function is constructed by the deviation between the projectile hole and the light-screen array measurement coordinates, and the flight data of the projectile is collected to form the blockchain, which is used for the inversion database during the machine learning process. The dimension of the inverse model is reduced by inputting all the internal parameters and ignoring the constant parameters, so that the efficiency of the algorithm is improved. The simulation result shows that the inverted external parameter values are close to the given true values, and the feasibility of the proposed method is verified by the live firing test.

In the next step, air resistance, gravity, and other factors can be considered to modify the trajectory; then, the data interpretation basis was introduced (i.e., PaTa Criterion) to reject the data with gross error before writing into the database, in order to make the inversion model more accurate.

Although applying the optimized model and the algorithm to the separated light-screen array will increase the dimension of structural parameters in the inversion model, they are still theoretically applicable. The results of this paper can provide a reference for the optimization of the light-screen array measurement model and the improvement of the precision, and the correlation algorithm can also provide reference for the identification of model parameters in other engineering problems.

\section{Data Availability}

The data used to support the findings of this study are included within the article.

\section{Conflicts of Interest}

The authors declare that they have no conflicts of interest.

\section{Acknowledgments}

This work was supported by the Scientific Research Plan Projects of Shaanxi Education Department (grant number 20JK0692).

\section{References}

[1] M. H. Ferdowsi and E. Sabzikar, "Optical target tracking by scheduled range measurements," Optical Engineering, vol. 54, no. 4, article 044101, 2015.

[2] N. A. Kazarinov, V. A. Bratov, N. F. Morozov et al., "Experimental and numerical analysis of PMMA impact fracture," International Journal of Impact Engineering, vol. 143, article 103597, 2020.

[3] A. Sedunov, A. Sutin, H. Salloum, and N. Sedunov, "Passive acoustic localization of small aircraft," Journal of the Acoustical Society of America, vol. 134, no. 5, p. 4076, 2013.

[4] Y. Li, Y. Wang, B. Liu, S. Zhang, L. Nie, and G. Bi, "A new motion parameter estimation and relocation scheme for airborne three-channel CSSAR-GMTI systems," IEEE Transactions on Geoscience and Remote Sensing, vol. 57, no. 6, pp. 4107-4120, 2019.

[5] W. H. Ma, T. Dong, H. Tian, and J. Ni, "Line-scan CCD camera calibration in 2D coordinate measurement," Optik, vol. 125, no. 17, pp. 4795-4798, 2014.

[6] H. S. Li and Z. Y. Lei, "Projectile two-dimensional coordinate measurement method based on optical fiber coding fire and its coordinate distribution probability," Measurement Science Review, vol. 13, no. 1, pp. 34-38, 2013.

[7] Z. C. Wu and X. L. Zhang, "On-sate calibration method of target distance of the sky screen target velocity measuring system," Optik, vol. 178, pp. 483-487, 2019.

[8] H. S. Li, Z. Lei, Z. Wang, and J. Gao, "Research object photoelectric characteristic and fire coordinate distributing probability in across screen system," Nanoelectronics and Optoelectronics, vol. 7, no. 2, pp. 199-203, 2012.

[9] J. P. Ni, Technology and Application of Measurement of the Light Screen Array, National Defense Industry Press, Peking, 2014.

[10] H. Tian, J. P. Ni, and M. X. Jiao, "Moment acquisition algorithm of a projectile passing through a trapezoidal screen," Acta Photonic Sinica, vol. 43, no. 12, article 1212001, 2014.

[11] R. Chen and J. P. Ni, "Optimization method of light-screearray structure parameters of photoelectric target based on orthogonal test," Acta Armamentarii, vol. 38, no. 11, pp. 2234-2239, 2017.

[12] Z. C. Wu, J. P. Ni, X. L. Zhang, and Y. Wu, "Study on verification device of screen spatial location parameters of sky screen target," Optik, vol. 125, no. 14, pp. 3770-3773, 2014.

[13] N. Deepa, Q. V. Pham, D. C. Nguyen et al., "A survey on blockchain for big data: approaches, opportunities, and future directions," ACM Computing Surveys, vol. 1, no. 1, 2020.

[14] H. Cui, Y. Guan, H. Chen, and W. Deng, "A novel advancing signal processing method based on coupled multi-stable stochastic resonance for fault detection," Applied Sciences, vol. 11, no. 12, p. 5385, 2021. 
[15] W. Deng, S. Shang, X. Cai et al., "Quantum differential evolution with cooperative coevolution framework and hybrid mutation strategy for large scale optimization," KnowledgeBased Systems, vol. 224, article 107080, 2021.

[16] X. Cai, H. Zhao, S. Shang et al., "An improved quantuminspired cooperative co-evolution algorithm with muli- strategy and its application," Expert Systems with Applications, vol. 171, article 114629, 2021.

[17] G. T. Reddy, M. P. K. Reddy, K. Lakshmanna et al., "Analysis of dimensionality reduction techniques on big data," IEEE Access, vol. 8, pp. 54776-54788, 2020. 